\title{
The Evolution of the Circumstellar and Interstellar Medium Around Massive Stars
}

\author{
S. Jane Arthur \\ Centro de Radioastronomía y Astrofísica, Universidad Nacional Autónoma de México, \\ Apartado Postal 3-72, 58090 Morelia, México \\ email: j.arthur@astrosmo.unam.mx
}

\begin{abstract}
Throughout their lives massive stars modify their environment through their ionizing photons and strong stellar winds. Here, I present coupled radiation-hydrodynamic calculations of the evolution of the bubbles and nebulae surrounding massive stars. The evolution is followed from the main sequence through the Wolf-Rayet stage and shows that structures are formed in the ISM out to some tens of parsecs radius. Closer to the star, instabilities lead to the breakup of swept-up wind shells. The photoevaporated flows from the resulting clumps interact with the stellar wind from the central star, which leads to the production of soft X-rays. I examine the consequences for the different observable structures at all time and size scales and evaluate the impact that the massive star has on its environment.
\end{abstract}

Keywords. stars: mass loss - ISM: bubbles - HII regions

\section{Introduction}

Massive stars interact with their environment both through their ionizing photons and their strong stellar winds. At early stages in their lives they produce bright emission-line nebulae such as the Trifid nebula and the Orion nebula. on size scales of up to a few parsecs. At the end of their lives, faint, photoionized $\mathrm{H} \alpha$ shells of tens of parsecs radius surround Wolf-Rayet (WR) stars.

Evidence for stellar wind bubbles around main sequence stars is not easy to come by because of the low density and high temperature of the shocked gas in the stellar wind bubble and confusion with the HII region. However, stellar wind shells have been detected kinematically (Nazé et al. 2001). The recent discovery of soft (2 million degrees), diffuse $\mathrm{X}$-rays in the Orion nebula (Güdel et al. 2008) presents a challenge to theorists. Diffuse $\mathrm{X}$-ray emission from star-forming regions had previously been detected at $\sim 10^{7} \mathrm{~K}$ in the Omega and Rosette nebulae, both of which surround clusters of massive stars (Dunne et al. 2003, Townsley et al. 2003). Stellar wind bubbles also manifest themselves as shells and holes of diameters of tens of parsecs in the distribution of HI gas around WR stars (e.g., Vasquez et al. 2005). Stellar wind bubbles of a few parsecs diameter are observed in optical emission lines around some WR stars and are distinguished from photoionized regions by their short dynamical timescales.

Traditional modeling of HII regions and stellar wind bubbles assumes a constant source of ionizing photons or stellar wind and a uniform ambient medium (Strömgren 1939, Kahn 1954, Dyson \& de Vries 1972, Weaver et al. 1977). The variation of stellar wind parameters as a function of stellar evolution was taken into account by García-Segura et al. (1996a,b) and the ionizing photon flux of the central star was included by Freyer et al. (2003, 2006). However, little attempt has been made to take into the account the effects of a non-uniform ambient medium during the initial stages. Another topic often mentioned but never modeled self-consistently is thermal conduction. Weaver et al. (1977) included 

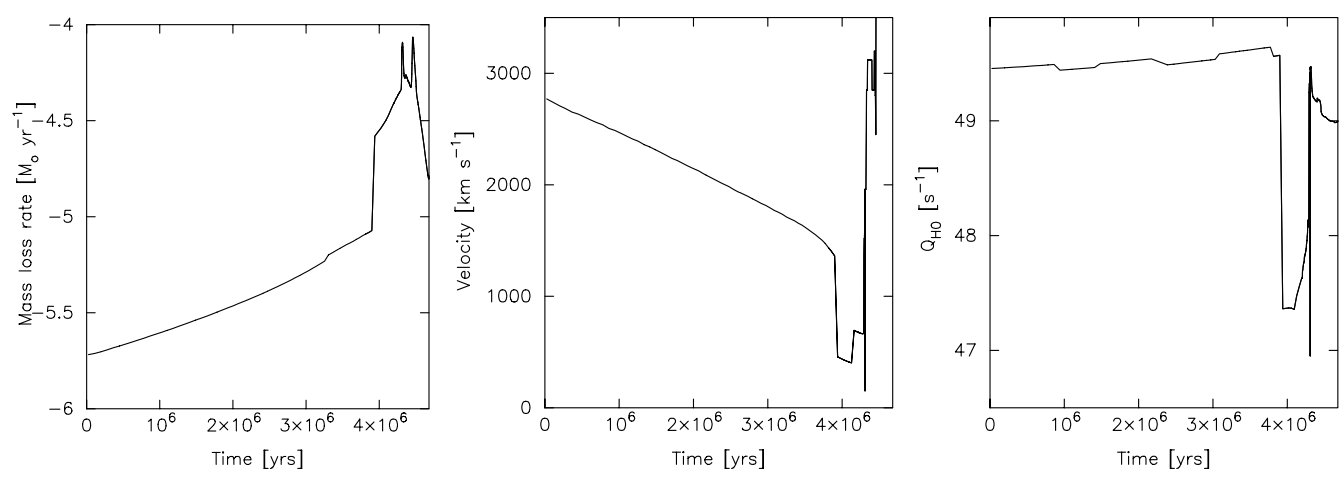

Figure 1. Mass-loss rate, stellar wind velocity and ionizing photon rate for a $60 \mathrm{M}_{\odot}$ star at solar metallicity and with an initial rotation velocity of $300 \mathrm{~km} \mathrm{~s}^{-1}$.

it in order to explain (mistakenly) the diffuse interstellar OVI emission detected with the Copernicus satellite. Theorists argue that the magnetic field in the swept-up shell will inhibit thermal conduction by electrons. In this paper we address both of these aspects.

\section{Modeling}

The variation of the stellar wind parameters with time can be found from stellar evolution models such as those of Meynet \& Maeder (2003). The most recent models, incorporating stellar rotation, suggest that such stars have double the WR lifetime of non-rotating models. The ionizing photon rate can be found from following the procedure outlined by Smith et al. (2002) (and included in the Starburst 99 code, Leitherer et al. 1999) using the appropriate values for effective temperature and stellar radius from the new stellar evolution models. In Figure 1 we show the mass-loss rate, stellar wind velocity and ionizing photon rate as a function of time for a $60 \mathrm{M}_{\odot}$ star of solar metallicity and initial rotation rate of $300 \mathrm{~km} \mathrm{~s}^{-1}$. It is clear that the star experiences periods of enhanced mass loss, when the stellar wind velocity and ionizing photon rate both fall dramatically.

It is also important to take into account the metallicity and ionization state of the gas when calculating the radiative cooling rate. Failure to do so will result in incorrect expansion speeds for the shells and bubble.

The ambient medium into which the HII region and stellar wind bubble expand is more difficult to define. In the initial stages, the star will still be embedded in the dense molecular cloud from which it formed. At some point, however, the photoionized region will break out of the cloud into the intercloud medium. Furthermore, many well-known WR stars have large proper motions, which indicates that they have moved a long way from their birthplace during their lifetime.

\section{HII regions in turbulent molecular clouds}

Massive stars are born in the dense cores of molecular clouds and so their initial evolution will take place in such an ambient medium. Molecular clouds are now thought to be clumpy as a result of supersonic turbulence due to colliding streams of gas. We have studied the structure and evolution of HII regions in such an environment using a state-of-the-art radiation hydrodynamics code (Mellema et al. 2006). As initial conditions we took the output of a 3D turbulence calculation (Vázquez-Semadeni et al. 2005). This $512^{3}$ grid represents a volume $4 \mathrm{pc}$ on a side. The average number density within the cube is $1000 \mathrm{~cm}^{-3}$ but the peak density in the clumps is $>10^{6} \mathrm{~cm}^{-3}$. The ionizing source is 

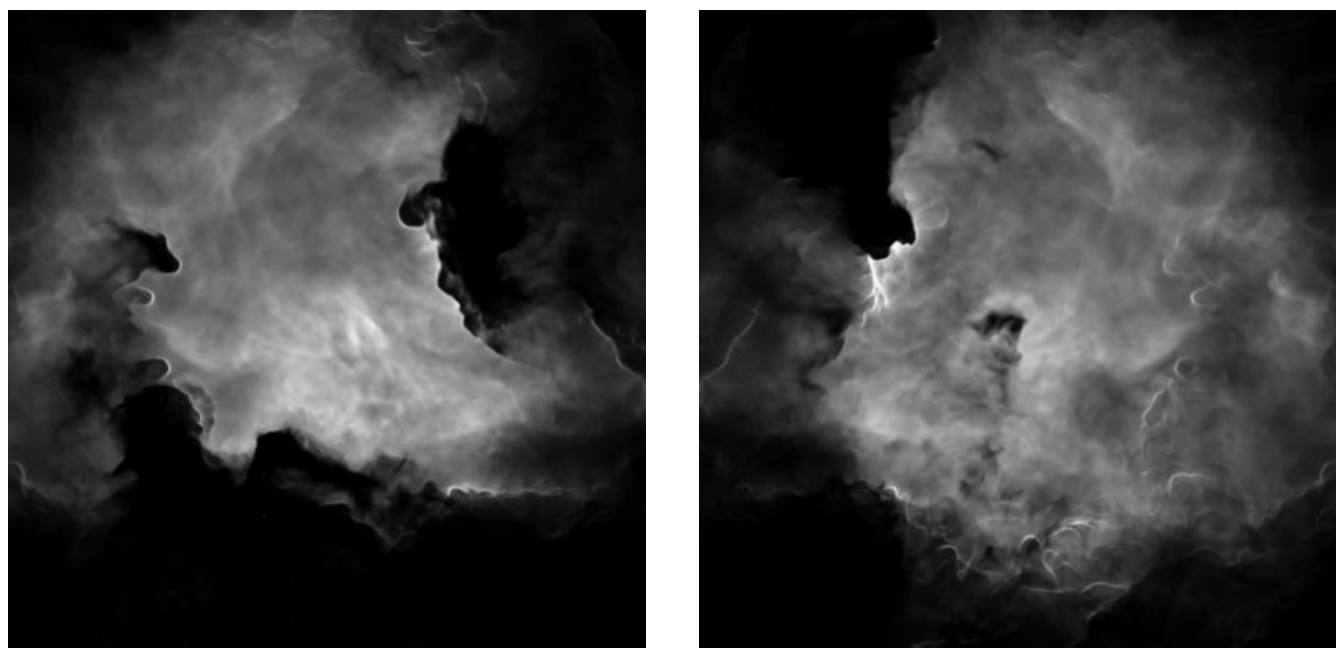

Figure 2. Synthetic optical emission-line image of a simulated HII region in a turbulent molecular cloud. Two views through the computational cube. Colors are the same as the standard HST filters-blue: OIII, green: $\mathrm{H} \alpha$, red: NII. Black regions represent foreground dust absorption.

located in the densest clump, which can be easily placed at the center of the grid because of the periodic boundary conditions of the turbulence calculation. The ionizing photon rate is set at $10^{48.5} \mathrm{~s}^{-1}$ for a star with effective temperature $37,000 \mathrm{~K}$.

Figure 2 shows a synthetic emission-line image after 250,000 yrs of evolution. The numerical simulation bears a remarkable resemblance to real HII regions like the Trifid nebula. Bright-rimmed fingers and columns show where the ionization front is eroding the dense neutral material. Photoevaporated flows from the tips of the fingers shock against each other in the inner part of the nebula leading to density and temperature enhancements there. Such simulations strongly suggest that the structure of young HII regions is mainly due to structure in the underlying molecular cloud rather than instabilities in the ionization front, as has been previously suggested (García-Segura \& Franco 1996).

The simulations described here do not yet include the stellar wind from the central star. This is principally due to the huge increase in the (already long) computational time required once the stellar wind is included because of the decrease in the timestep that this entails (Arthur \& Hoare 2006). It is interesting that the main features of real HII regions can be produced with our simulations without stellar winds for these early stages in the life of a massive star.

\section{Stellar wind bubbles and HII regions}

At some point the stellar wind will become important and the swept-up shell of material driven by the hot shocked stellar wind bubble will trap the ionization front. The trapped HII region expands with time since its temperature is fixed at $\sim 10^{4} \mathrm{~K}$ and the pressure is set by the hot shocked bubble. Moreover, the ionization front requires a shock in the neutral gas ahead of it and so the stellar wind bubble is surrounded by a thick shell of shocked neutral material.

In Figure 3 we show the variation of density and temperature of the gas with radius in the stellar wind bubble towards the end of the main-sequence stage of the $60 \mathrm{M}_{\odot}$ star for cases with and without time-dependent thermal conduction. The stellar parameters are those depicted in Figure 1. This is a spherically symmetric calculation in order to 

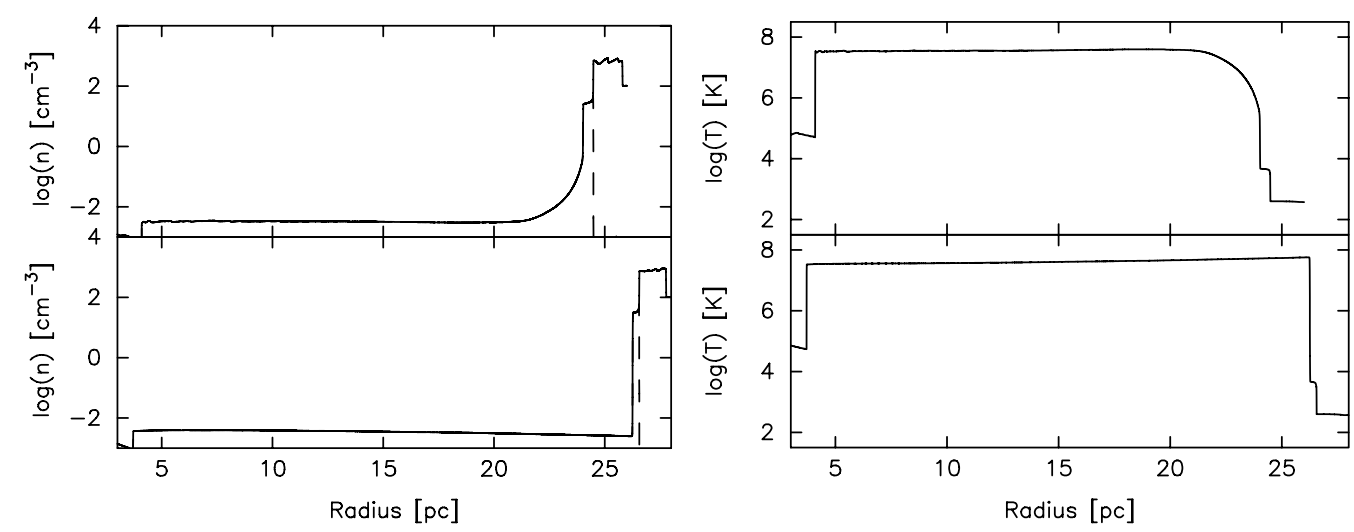

Figure 3. Density and temperature distributions of a main sequence stellar wind bubble. Top: With time-dependent thermal conduction. Bottom: Without thermal conduction. Solid line: total number density. Dashed line: Ionized number density, showing limit of HII region.

be able to follow several million years of stellar evolution and we have assumed that the ambient medium is uniform and neutral with a number density $n_{0}=100 \mathrm{~cm}^{-3}$ and temperature $\sim 500 \mathrm{~K}$ representing the photodissociated region ahead of the ionization front. The stellar wind is modeled as a region of uniform mass and energy deposition (e.g., Chevalier \& Clegg 1985) since this treatment makes it easier to follow changes in the stellar wind. The resulting thermal wind becomes supersonic well before the inner stellar wind shock, hence the details of the wind treatment have no effect on the formation and expansion of the stellar wind bubble.

We see that thermal conduction enhances the density in the hot shocked bubble close to its edge. The increased cooling rate here lowers the temperature at the edge of the bubble and the average pressure in the bubble decreases. This slows the expansion rate and also allows the stellar wind shock to move outwards further from the star than in the corresponding case without thermal conduction.

When the stellar wind velocity drops during a period of enhanced mass loss, such as a red supergiant or LBV stage, the ram pressure of the stellar wind is much less than the thermal pressure in the hot shocked bubble and so the bubble begins to back fill. Moreover, when the ionizing photon rate becomes so low that it cannot sustain the HII region at the edge of the stellar wind bubble, the gas here recombines. This has the consequence that the pressure here drops to half its previous value, and so the shocked neutral material expands into this region. When the ionizing photon rate increases again in a Wolf-Rayet phase, the HII region reforms at the edge of the stellar wind bubble.

\section{Instabilities}

The slow, dense wind material of a red supergiant or LBV stage is squeezed into a thin shell by the high pressure of the surrounding hot shocked bubble. This thin, dense, neutral shell is subject to a variety of instabilities such as the thin shell instability and Rayleigh-Taylor instabilities, which break it up into dense neutral clumps, as shown in Figure 4. Once the ionizing photon rate increases again, these clumps acquire photoionized skins and the resulting transonic, dense, photoevaporated flows shock against the stellar wind, leading to densities and temperatures that should be observable in X-rays. In this scenario, the optical emission would come from the shocked neutral shell material, while the X-ray emission is produced by the shocked photoevaporated flows. 

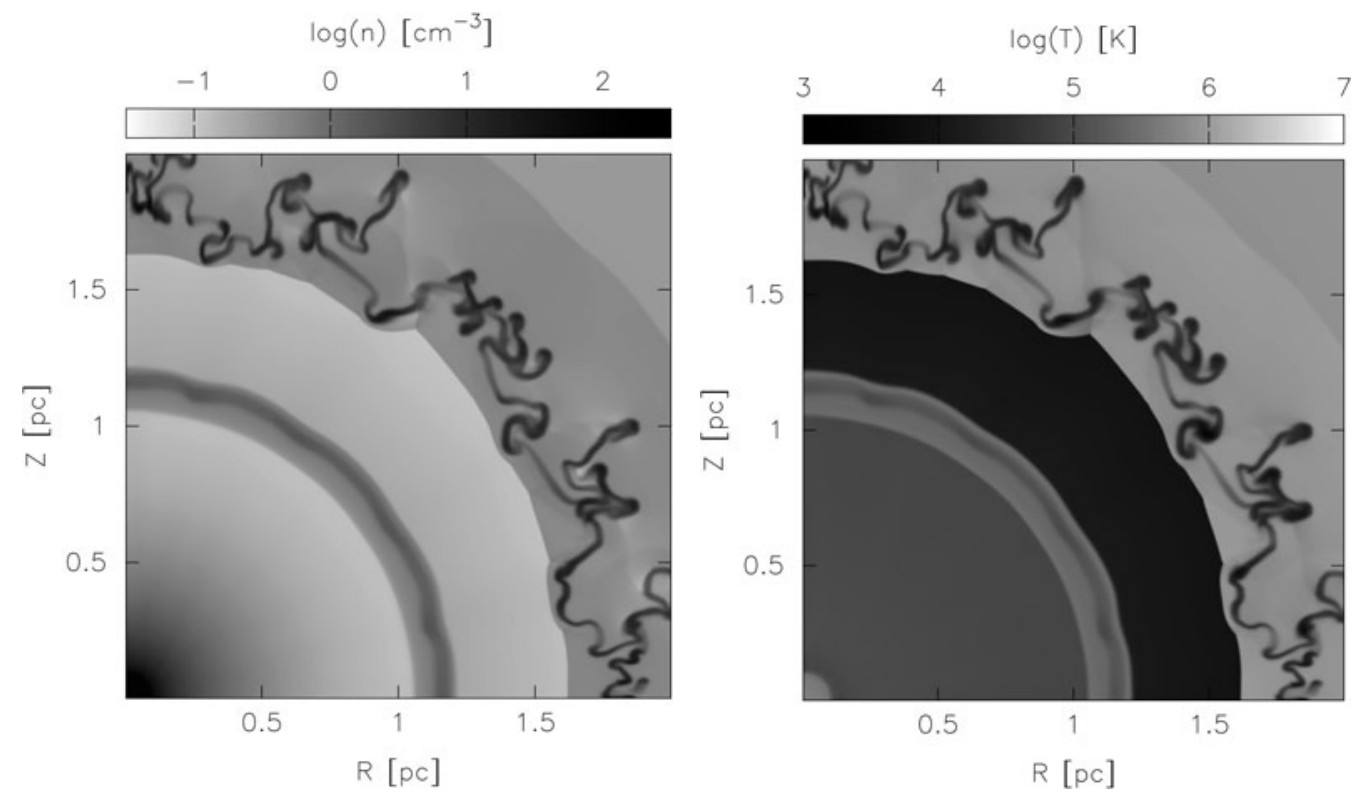

Figure 4. Interaction of a fast Wolf-Rayet wind with a dense red supergiant wind shell. Left: Ionized number density. Right: Temperature.
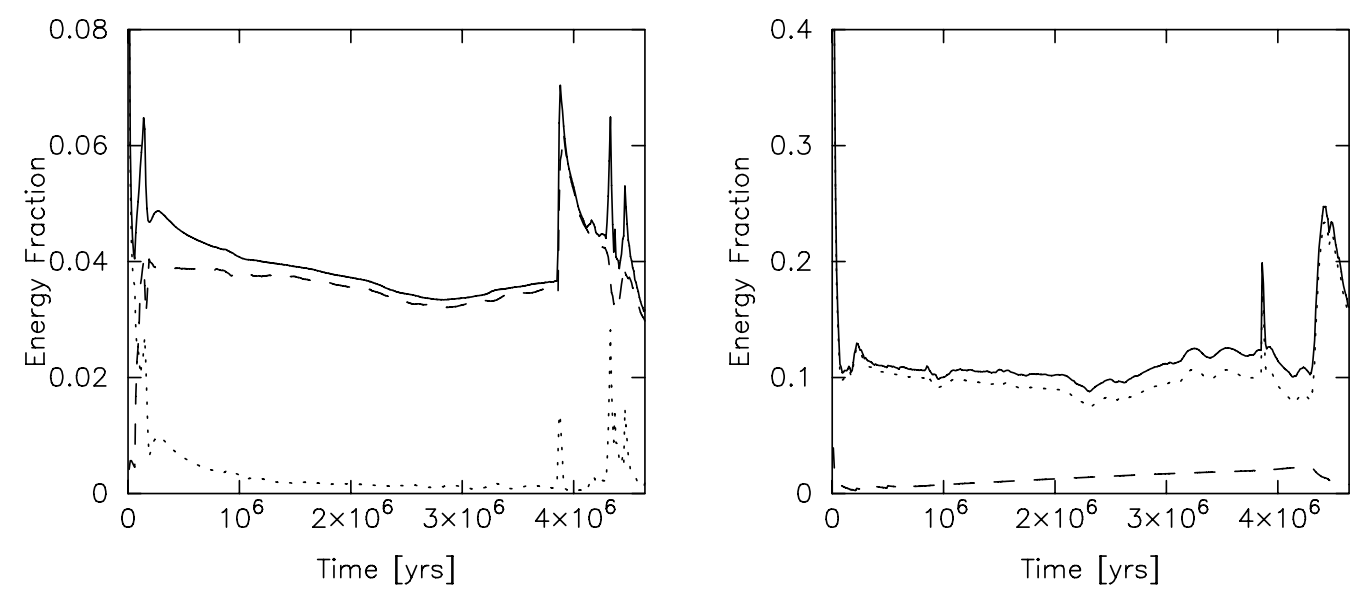

Figure 5. Left: Gas kinetic energy as a fraction of total wind mechanical energy for the model with time-dependent thermal conduction. Right: Gas thermal energy. Solid line: Total gas kinetic (thermal) energy. Dashed line: Neutral gas kinetic (thermal) energy. Dotted line: Ionized gas kinetic (thermal) energy. Note the different scales on the two graphs.

\section{Energy budget}

Observational studies of the HI shells around some Galactic WR stars (Vasquez et al. 2005) and also of the superbubble N51D in the LMC (Cooper et al. 2004) reveal that only a small fraction of the total stellar wind (and supernova) mechanical energy is imparted to the interstellar medium and argue that this constitutes an energy crisis for these bubbles. However, the present calculations show that even though the gas thermal and kinetic energies are a small fraction of the total stellar wind energy over the star's lifetime (see Fig. 5), the explanation for the 'missing' energy is simply that it was radiated away. 


\section{Summary}

Radiation-hydrodynamic modeling shows how different structures form and evolve in the circumstellar and interstellar medium around massive stars. Structure in optical HII regions results from differences in densities and hence opacities in an underlying clumpy medium. Main-sequence stellar wind bubbles are surrounded by HII regions and thick neutral shells, making their direct detection difficult. Thermal conduction, if present, leads to enhanced radiative energy losses at the edge of the hot, shocked wind bubble, reducing its pressure and slowing the expansion. The interaction of the fast wind from the WR stage with a dense neutral shell from a previous red supergiant or LBV stage leads to instabilities, which break up the neutral shell into clumps.

The amount of energy imparted to the interstellar medium as kinetic or thermal energy of the gas is only a small fraction of the total stellar wind energy released over the star's lifetime, with the remainder of the energy simply being radiated away. A single massive star can affect the structure and dynamics of the interstellar medium at distances of up to tens of parsecs and the compression and subsequent fragmentation of dense shells can lead to new episodes of star formation.

\section{References}

Arthur, S. J., \& Hoare, M. G. 2006, ApJS, 165, 283

Chevalier, R. A., \& Clegg, A. W. 1985, Nature, 317, 44

Cooper, R. L., Guerrero, M. A., Chu, Y.-H., et al. 2004, ApJ, 605, 751

Dunne, B. C., Chu, Y.-H., Chen, C.-H. R., et al. 2003, ApJ, 590, 306

Dyson, J. E., \& de Vries, J. 1972, A\& A, 20, 223

Freyer, T., Hensler, G., \& Yorke, H. W. 2003, ApJ, 594, 888

Freyer, T., Hensler, G., \& Yorke, H. W. 2006, ApJ, 638, 262

García-Segura, G. \& Franco, J. 1996, ApJ, 469, 171

García-Segura, G., Mac Low, M.-M., \& Langer, N. 1996a, A\&A, 305, 229

García-Segura, G., Langer, N., \& Mac Low, M.-M. 1996b, A\&A, 316, 133

Güdel, M., Briggs, K. R., Montmerle, T., et al. 2008, Sci, 319, 309

Kahn, F. D. 1954, Bull. Astron. Inst. Netherlands, 12, 187

Leitherer, C., et al. 1999, ApJS, 123, 3

Mellema, G., Arthur, S. J., Henney, W. J., Iliev, I. T., \& Shapiro, P. R. 2006, ApJ, 647, 397

Meynet, G., \& Maeder, A. 2003, A\& A, 404, 975

Nazé, Y., Chu, Y.-H., Points, S. D., et al. 2001, AJ, 122, 921

Smith, L. J., Norris, R. P. F., \& Crowther, P. A. 2002, MNRAS, 337, 1309

Strömgren, B. 1939, ApJ, 89, 526

Townsley, L. K., Feigelson, E. D., Montmerle, et al. 2003, ApJ, 593, 874

Vasquez, J., Cappa, C., \& McClure-Griffiths, N. M. 2005, MNRAS, 362, 681

Vázquez-Semadeni, E., Kim, J., Shadmehri, M., \& Ballesteros-Paredes, J. 2005, ApJ, 618, 344

Weaver, R., McCray, R., Castor, J., et al. 1977, ApJ, 218, 377

\section{Discussion}

WALBORN: It's very interesting that you get the pillars with just the radiation and no winds. Do you have any indications of the effects of the winds on the formation (or disruption?) of these structures?

ARTHUR: The pillars are the result of the interaction of the ionizing photons with the dense clumps in the molecular cloud and show the position of the ionization front. A stellar wind bubble would be internal to the ionization front and, in regions where there is a density gradient, would tend to blow out in the direction away from the dense cloud. I don't think a wind would make any difference to the formation of these structures. 